\title{
Accurate Noise Floor Calibration based on Modified Expectation Maximisation of Gaussian Mixture
}

\author{
Miguel López-Benítez ${ }^{\dagger \ddagger}$, Janne Lehtomäki*, Kenta Umebayashi ${ }^{\S}$, Dhaval Patel ${ }^{\uparrow}$ \\ ${ }^{\dagger}$ Department of Electrical Engineering and Electronics, University of Liverpool, United Kingdom \\ $\ddagger$ ARIES Research Centre, Antonio de Nebrija University, Spain \\ *Centre for Wireless Communications, University of Oulu, Finland

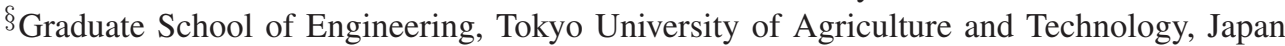 \\ ${ }^{\top}$ School of Engineering and Applied Science, Ahmedabad University, India \\ Email: M.Lopez-Benitez@liverpool.ac.uk
}

\begin{abstract}
An accurate estimation of the noise floor is of paramount importance for an optimum performance of spectrum sensing in Cognitive Radio (CR). The most common approach followed by existing noise floor estimation methods is to attempt to isolate a set of noise-only samples based on a given energy/power threshold. However, this approach is unreliable and in general unable to provide accurate estimations of the noise floor, in particular under low SNR conditions where the power of the Primary User (PU) signal is comparable to the noise floor of the CR device. In this context, this work considers a different approach where the power observed by the CR device is modelled as a Gaussian mixture. Based on a mathematical analysis of the relation among the parameters of the obtained Gaussian mixture, a modified version of the well-known Expectation Maximisation (EM) algorithm is proposed to fit the Gaussian mixture to the observed power values and provide an estimation of the noise floor, something that the general EM algorithm fails to achieve in this scenario. The obtained results demonstrate that the proposed method provides a highly accurate estimation of the noise floor in the presence of PU signals over the whole range of SNR values.
\end{abstract}

\section{INTRODUCTION}

Cognitive Radio (CR) devices are required to operate under strict interference constraints, which translates into demanding Primary User (PU) signal detection requirements [1]. A broad range of methods for signal detection, referred to as spectrum sensing in CR jargon, have been proposed in the literature [2]. The performance of spectrum sensing algorithms is severely constrained by the noise floor of the $\mathrm{CR}$ receiver, which is defined as the aggregated power of all internal noise sources and external unwanted signals. The noise floor determines the minimum PU signal level that can be reliably detected by a CR device. A well-known example is the case of energy detection, which determines the presence of a PU signal by comparing the energy (or power) of the observed signal samples with a predefined decision threshold. Such decision threshold is frequently selected based on the receiver's noise floor so as to guarantee a desired constant false alarm rate [3], although it can be selected based on other criteria that usually depend on the receiver's noise floor as well [4]. One of the main and wellknown phenomena that degrade the performance of energy detection is the variability (uncertainty) of the noise floor, which imposes a fundamental limit on the minimum Signalto-Noise Ratio (SNR) below which the PU signal cannot be reliably detected (known as SNR wall [5]). An accurate estimation of the noise floor can help to overcome the SNR wall and is therefore of paramount importance to guarantee an optimum performance of spectrum sensing in CR.

The noise floor of a receiver is not static and therefore a periodic estimation is required [6]. The aggregated power of the internal noise sources could be readily estimated with a hardware implementation where the CR device can switch from the antenna port to a matched load in order to obtain clean samples of the internal noise [7]. However, this approach would not be useful in practice because the effective noise floor would increase when the receiver switches back to the antenna for normal operation as a result of external noise sources such as ambient noise, man-made noise or out-of-band transmissions [8]. Since these external sources of noise must be taken into account, the $\mathrm{CR}$ receiver needs to estimate the noise floor while connected to an antenna, which makes the presence of PU signals unavoidable. The main challenge faced in practical implementations is the accurate estimation of the noise floor in the presence of PU signals. While this is not a new problem [9] and several approaches have been proposed, existing solutions suffer from drawbacks and limitations.

Most existing methods [10]-[13] rely on a threshold-based classification of the observed energy/power samples into two sets, one that is assumed to contain noise-only samples (i.e., samples of the observed energy/power when the PU is assumed to be absent) and another one that is assumed to contain signalplus-noise samples (i.e., samples of the observed energy/power when the PU is assumed to be present). The samples in the first set (i.e., the assumed noise-only samples) are then used to estimate the noise floor. In practice it is nearly impossible to perform such classification in a truly reliable way, in particular under low SNR conditions where the PU signal energy/power level is comparable to that of the CR receiver's noise floor. As a result, this type of methods is unable to produce a truly clean set of noise-only samples that can be used to provide an accurate estimation of the noise floor and, as a matter of fact, can sometimes fail catastrophically [14].

In this context, this work explores a different approach based on the appreciation that the observed energy/power samples are distributed according to a weighted sum of Gaussian dis- 
tributions (i.e., Gaussian mixture). Based on this observation, the noise floor can be estimated by fitting the expression of the Gaussian mixture to a set of energy/power samples. However, the well-know Expectation Maximisation (EM) algorithm that is commonly employed to perform such fit is unable (as it will be shown) to provide satisfactory results. This limitation of the general EM algorithm motivates this work, where a modified version is proposed. The main difference of the proposed EM approach is the explicit inclusion into the fitting process of a key mathematical relation among the parameters of the considered Gaussian mixture, which enables the EM method to provide a highly accurate estimation of the noise floor in the presence of PU signals over the whole SNR range.

The rest of this work is organised as follows. First, Section II presents the considered system model and provides a formal description of the problem under study. Some threshold-based classification methods are discussed in Section III, while the general and proposed EM approaches are presented in Section IV. The performance of the considered methods is assessed in Section V. Finally, Section VI concludes this work.

\section{System Model And PRoblem Formulation}

In order to estimate the noise floor, the CR device records a sufficiently large set of $K$ samples of the observed signal power $\mathbf{P}=\left\{\mathcal{P}_{1}, \ldots, \mathcal{P}_{k}, \ldots, \mathcal{P}_{K}\right\}=\left\{\mathcal{P}_{k}\right\}_{k=1}^{K}$ obtained as:

$$
\mathcal{P}_{k}=\frac{1}{N} \sum_{n=1}^{N}|y[n]|^{2}
$$

where $y[n]$ denotes the observed signal samples and $N$ is the signal sample size over which the power is estimated.

The observed signal samples can be expressed as:

$$
y[n]= \begin{cases}w[n], & \mathcal{H}_{0} \text { (PU signal is absent) } \\ x[n]+w[n], & \mathcal{H}_{1} \text { (PU signal is present) }\end{cases}
$$

where $w[n] \sim \mathcal{C N}\left(0, \sigma_{w}^{2}\right)$ represents the complex baseband samples of the noise at the CR device (including internal noise sources and external unwanted signals) distributed according to a Circularly Symmetric Complex Gaussian (CSCG) distribution with zero mean and variance/power equal to $\sigma_{w}^{2}$, while $x[n] \sim \mathcal{C N}\left(0, \sigma_{x}^{2}\right)$ denotes the complex baseband samples of the received PU signal, assumed to follow a CSCG distribution with zero mean and variance/power equal to $\sigma_{x}^{2}$.

According to the central limit theorem, the power samples $\mathcal{P}_{k}$ in (1) can be assumed to be normally distributed with a certain mean and variance. When the PU signal is absent $\left(\mathcal{H}_{0}\right)$, $\mathcal{P}_{k} \sim \mathcal{N}\left(\mu_{0}, \sigma_{0}^{2}\right)$ with mean $\mu_{0}$ and variance $\sigma_{0}^{2}$ given by:

$$
\begin{aligned}
& \mu_{0}=\mathrm{E}\left(\mathcal{P}_{k} \mid \mathcal{H}_{0}\right)=\sigma_{w}^{2} \\
& \sigma_{0}^{2}=\operatorname{Var}\left(\mathcal{P}_{k} \mid \mathcal{H}_{0}\right)=\frac{\sigma_{w}^{4}}{N}
\end{aligned}
$$

However, when a PU signal is present $\left(\mathcal{H}_{1}\right), \mathcal{P}_{k} \sim \mathcal{N}\left(\mu_{1}, \sigma_{1}^{2}\right)$ with mean $\mu_{1}$ and variance $\sigma_{1}^{2}$ given by:

$$
\begin{aligned}
\mu_{1} & =\mathrm{E}\left(\mathcal{P}_{k} \mid \mathcal{H}_{1}\right)=\sigma_{x}^{2}+\sigma_{w}^{2} \\
\sigma_{1}^{2} & =\operatorname{Var}\left(\mathcal{P}_{k} \mid \mathcal{H}_{1}\right)=\frac{\left(\sigma_{x}^{2}+\sigma_{w}^{2}\right)^{2}}{N}
\end{aligned}
$$

Note than when a PU signal is present, the SNR $\gamma=\sigma_{x}^{2} / \sigma_{w}^{2}$ can be expressed as a function of these parameters as:

$$
\gamma=\frac{\mu_{1}}{\mu_{0}}-1=\sqrt{\frac{\sigma_{1}^{2}}{\sigma_{0}^{2}}}-1
$$

In a channel with intermittent on/off usage from a single PU, the Probability Density Function (PDF) of the power observed by a $\mathrm{CR}$ device can be characterised as:

$$
f_{\mathcal{P}_{k}}(x)=(1-\Psi) \mathcal{N}\left(x \mid \mu_{0}, \sigma_{0}^{2}\right)+\Psi \mathcal{N}\left(x \mid \mu_{1}, \sigma_{1}^{2}\right)
$$

where $\Psi=P\left(\mathcal{H}_{1}\right)$ denotes the probability that the PU signal is present (i.e., the duty cycle of the PU channel). While the model in (8) assumes that a single PU with signal power $\sigma_{x}^{2}$ is present in the channel, it can be extended to an arbitrary number of $M$ disjoint PUs with different transmission powers:

$$
f_{\mathcal{P}_{k}}(x)=\sum_{m=0}^{M} \omega_{m} \cdot \mathcal{N}\left(x \mid \mu_{m}, \sigma_{m}^{2}\right)
$$

where the weights $\omega_{m}$ satisfy the conditions $\omega_{m} \in(0,1)$ and $\sum_{m=0}^{M} \omega_{m}=1$. The single PU model in (8) can be seen as a particular case of (9) with $M=1$ (i.e., only one PU), $\omega_{0}=$ $1-\Psi$ and $\omega_{1}=\Psi$. For the sake of simplicity and without loss of generality, the single PU model in (8) will be considered in this work. In a practical context, the number of PUs present in the channel (and therefore the required number of Gaussian components, $M+1$ ) can be determined for example based on the Bayesian information criterion as detailed in [15].

The problem addressed in this work is how to estimate accurately the noise floor (i.e., the average noise power $\mu_{0}=\sigma_{w}^{2}$ ) based on a set $\mathbf{P}$ of $K$ empirically observed power samples.

\section{Threshold-BASED ClASSIFICATION METHODS}

This type of methods estimate the noise floor from a subset $\mathbf{P}_{\mathbf{0}} \subseteq \mathbf{P}$ assumed to contain only noise power samples (i.e., power samples observed in the absence of a PU signal). This set is obtained by defining a power threshold $\lambda$ and assuming that all the power samples below that threshold correspond to noise-only samples, i.e., $\mathbf{P}_{\mathbf{0}}=\left\{\mathcal{P}_{k}: \mathcal{P}_{k} \leq \lambda\right\}$. The main difference among the existing threshold-based methods is how the threshold $\lambda$ is set. Some examples are discussed below.

The Otsu method [10] was proposed in the field of image processing to automatically reduce graylevel images to binary images. This method has also been proposed for the processing of empirical spectrum measurements in the context of $\mathrm{CR}$ to determine when a PU signal is present based on observed power samples [7]. The Otsu threshold is calculated so as to minimise the intra-class variance (i.e., between the power samples classified as either $\mathcal{H}_{0}$ or $\mathcal{H}_{1}$ ), which is shown to be equivalent to maximise the inter-class variance (i.e., variance of the power samples classified within the same set).

More recently, the Forward Consecutive Mean Excision (FCME) method [12] and some variants thereof [14] have been proposed to the same end. This method first sorts the values of the power samples in the observed set $\mathbf{P}$ in ascending order. 
The algorithm starts with a small initial subset of samples (typically the 10th percentile) and calculates its mean value (initial $\left.\mu_{0}\right)$, which is used to calculate the threshold as $\lambda=\mu_{0} \cdot T_{C M E}$, where $T_{C M E}$ is a configurable parameter. A new value for the mean $\mu_{0}$ is calculated from the samples below the previous threshold $\lambda$. The FCME algorithm iteratively recalculates new values for the mean and the corresponding threshold until there are no more new power samples below the threshold. The mean value $\mu_{0}$ from the last iteration is the estimated noise floor. The $T_{C M E}$ parameter determines the accuracy and convergence speed of the algorithm and is selected based on the desired Clean Sample Rejection Rate (CSRR), which is the fraction of noise-only power samples $\left(\mathcal{H}_{0}\right)$ that are incorrectly classified as samples with signal components $\left(\mathcal{H}_{1}\right)$. The value of this parameter can be calculated as [16, eq. (12)]:

$$
T_{C M E}=F^{-1}(1-\mathrm{CSSR}, 2 N, 2 N(K-1))
$$

where $F^{-1}(\cdot)$ denotes the inverse of the $F$ (Fisher) cumulative distribution function [17, eq. (26.6.1)].

The main limitation of threshold-based classification algorithms is the inability to perform a reliable classification of the observed power samples $\mathbf{P}$ into two sets for $\mathcal{H}_{0}$ and $\mathcal{H}_{1}$. Under high SNR conditions $\left(\mu_{1} \gg \mu_{0}\right)$, the power samples drawn from $\mathcal{N}\left(\mu_{0}, \sigma_{0}^{2}\right)$ and $\mathcal{N}\left(\mu_{1}, \sigma_{1}^{2}\right)$ are sufficiently apart to allow for a wide range of values of $\lambda$ that can lead to a perfect classification. However, as the SNR decreases, the power samples drawn from both distributions (i.e., observed under the presence and absence of a PU signal) become similar and it is impossible to distinguish both in a reliable manner. As a result, the assumed noise-only samples will not always be so and the noise floor estimated from such set will be inaccurate.

\section{Expectation MAXimis ation Methods}

An alternative approach to estimate the noise floor is to fit the distribution in (9) to the elements of the set $\mathbf{P}$, which means estimating the parameters $\boldsymbol{\theta}=\left\{\omega_{m}, \mu_{m}, \sigma_{m}^{2}\right\}_{m=0}^{M}$ from the set $\mathbf{P}$ of empirically observed power samples. In addition to the parameter of interest in this work (i.e., the noise floor $\left.\mu_{0}\right)$, this process also provides an estimation of other relevant parameters such as the channel duty cycle $(\Psi)$ or the SNR, which can be obtained based on (7).

A Gaussian distribution can be fitted to empirical samples through Maximum Likelihood Estimation (MLE) of the mean and variance. However, in the case of a weighted sum of multiple Gaussian distributions (Gaussian mixture) as shown in (9), the MLE equations cannot be solved analytically. While some methods have been proposed to solve these equations numerically, the EM method is certainly the most popular one.

\section{A. General Expectation Maximisation Method}

The EM method starts with an initial guess of the parameters to be estimated $\boldsymbol{\theta}=\left\{\omega_{m}, \mu_{m}, \sigma_{m}^{2}\right\}_{m=0}^{M}$, which can be chosen randomly or following some heuristic method (e.g., using the $k$-means algorithm to cluster the data set $\mathbf{P}$ and then select the initial guess $\boldsymbol{\theta}$ based on $k$-means memberships). The EM method then finds numerically the MLE of the parameters of the Gaussian mixture based on an iterative process.

Each iteration of the EM method consists of an expectation (E) step and a maximisation (M) step. In the original method proposed in [18], the E-step calculates the expected value of the log-likelihood function using the last estimates of the model parameters $\boldsymbol{\theta}$ (or an initial guess in the first iteration). However, referring nowadays to this step as expectation is certainly a misnomer since the method was modified to compute the maximum a posteriori estimates for Bayesian inference. In the modern version of the method, the E-step calculates the posteriori probabilities $p\left(m \mid \mathcal{P}_{k}\right)$ of each Gaussian component $m$ for each observed power sample $\mathcal{P}_{k}$. These probabilities, referred to as membership probabilities, are calculated based on the last estimates of the model parameters $\boldsymbol{\theta}$ (or an initial guess in the first iteration) as follows:

$$
p\left(m \mid \mathcal{P}_{k}\right)=\frac{\omega_{m} \cdot \mathcal{N}\left(\mathcal{P}_{k} \mid \mu_{m}, \sigma_{m}^{2}\right)}{\sum_{m=0}^{M} \omega_{m} \cdot \mathcal{N}\left(\mathcal{P}_{k} \mid \mu_{m}, \sigma_{m}^{2}\right)}
$$

The M-step then computes new MLE estimates of the parameters $\boldsymbol{\theta}$ by maximising the expected log-likelihood function based on the probabilities $p\left(m \mid \mathcal{P}_{k}\right)$ obtained from the E-step:

$$
\begin{aligned}
\omega_{m} & =\frac{\sum_{k=1}^{K} p\left(m \mid \mathcal{P}_{k}\right)}{K} \\
\mu_{m} & =\frac{\sum_{k=1}^{K} p\left(m \mid \mathcal{P}_{k}\right) \cdot \mathcal{P}_{k}}{\sum_{k=1}^{K} p\left(m \mid \mathcal{P}_{k}\right)} \\
\sigma_{m}^{2} & =\frac{\sum_{k=1}^{K} p\left(m \mid \mathcal{P}_{k}\right) \cdot\left(\mathcal{P}_{k}-\mu_{m}\right)^{2}}{\sum_{k=1}^{K} p\left(m \mid \mathcal{P}_{k}\right)}
\end{aligned}
$$

The process is repeated until convergence is detected, which is determined by computing the log-likelihood function:

$$
\mathcal{L}(\boldsymbol{\theta} ; \mathbf{P})=\sum_{k=1}^{K} \ln \left[\sum_{m=0}^{M} \omega_{m} \cdot \mathcal{N}\left(\mathcal{P}_{k} \mid \mu_{m}, \sigma_{m}^{2}\right)\right]
$$

and comparing its value in the current iteration $\mathcal{L}_{\text {new }}(\boldsymbol{\theta} ; \mathbf{P})$ with that of the previous iteration $\mathcal{L}_{\text {old }}(\boldsymbol{\theta} ; \mathbf{P})$. The iterative process ends when the variation in the value of the loglikelihood function between consecutive iterations is below a predefined tolerance threshold $\varepsilon$ :

$$
\left|\frac{\mathcal{L}_{\text {new }}(\boldsymbol{\theta} ; \mathbf{P})-\mathcal{L}_{\text {old }}(\boldsymbol{\theta} ; \mathbf{P})}{\mathcal{L}_{\text {old }}(\boldsymbol{\theta} ; \mathbf{P})}\right| \leq \varepsilon
$$

When (16) is satisfied, the estimated parameters of the Gaussian mixture, $\boldsymbol{\theta}$, are assumed to converge to fixed values.

\section{B. Proposed Expectation Maximisation Method}

While the EM method is guaranteed to converge, it does not necessarily converge to the global optimum of (15). In fact, the EM method is known to converge typically to local optima. When this method is employed to fit the Gaussian mixture in (8) to a set of empirical power samples $\mathbf{P}$, the obtained result is often inaccurate as it will be shown in Section V.

A variety of solutions can be used to overcome this problem. For example, since the point to which the EM method converges depends on the initial guess of the model parameters $\boldsymbol{\theta}$, 
it may be possible to escape a local maximum by restarting the algorithm with different random initial guesses or by applying simulated annealing methods. However, this type of heuristic approaches are inelegant and increase the complexity.

This paper proposes an alternative, much simpler solution based on the insights gained from the mathematical analysis of the relations among the parameters of the Gaussian mixture in (8)-(9). Notice that, in the most general case, the parameters of the Gaussian distribution (i.e., its mean and variance) are in general mutually independent (i.e., their values are unrelated). The general EM method assumes this general case where the mean and variance of the distribution are independent and therefore estimates both parameters independently from the sample set based on (13) and (14), respectively. However, in the Gaussian mixture considered in this work, the inspection of (3)-(6) reveals that mean and variance are not independent but related in closed form as:

$$
\sigma_{m}^{2}=\frac{\mu_{m}^{2}}{N}
$$

Therefore, only one parameter (either mean or variance) needs to be estimated - the other one can be obtained using (17). In general, an accurate sample estimation of higher order moments requires a larger sample size. Conversely, given a finite sample set, the sample estimates of lower order moments are in general more accurate. Therefore, a more accurate fit of (8)-(9) to $\mathbf{P}$ can be expected if the means $\left\{\mu_{m}\right\}_{m=0}^{M}$ (first raw moments) are first estimated from the sample set using (13) and the variances $\left\{\sigma_{m}^{2}\right\}_{m=0}^{M}$ (second central moments) are then estimated using (17). The proposed modified version of the EM method is thus obtained by replacing (14) with (17). While this is a minor modification of the algorithm, the impact on its accuracy is very significant as it will be shown.

\section{Simulation Results}

The accuracy of the proposed EM method was assessed and compared with the rest of estimation methods considered in this work by means of simulations. The noise floor was set to a fixed value calculated as $\mu_{0}=\sigma_{w}^{2}=k_{B} T B F$, where $k_{B}=1.38064852 \cdot 10^{-23} \mathrm{~J} \cdot \mathrm{K}^{-1}$ is the Boltzmann constant, $T=290 \mathrm{~K}$ is the room temperature, $B=1 \mathrm{MHz}$ is the receiving bandwidth of the $\mathrm{CR}$ device, and $F=10$ is its noise factor (equivalent to a noise figure of $10 \mathrm{~dB}$ ). This leads to a noise floor of approximately $4 \cdot 10^{-14} \mathrm{~W}$ or $-104 \mathrm{dBm}$. A single PU was assumed to be present in the channel with an activity factor (duty cycle) equal to $\Psi=0.3$ and a transmission power equal to $\sigma_{x}^{2}=\gamma \sigma_{w}^{2}$, which was adjusted to simulate a range of SNR values from $-30 \mathrm{~dB}$ to $+5 \mathrm{~dB}$. A set $\mathbf{P}$ of $K=10^{5}$ power samples (assumed to be estimated based on blocks of $N=100$ signal samples) was randomly generated. The selected sample size $\left(K=10^{5}\right)$ was observed to be sufficiently large to allow all the considered estimation methods provide their best attainable accuracy and thus remove any potential bias resulting from a limited amount of samples. Out of these $K$ samples, $(1-\Psi) K=7 \cdot 10^{4}$ samples were drawn from a Gaussian distribution $\mathcal{N}\left(\mu_{0}, \sigma_{0}^{2}\right)$ corresponding to power samples observed in the absence of the PU signal, while the remaining $\Psi K=3 \cdot 10^{4}$ samples were drawn from a Gaussian distribution $\mathcal{N}\left(\mu_{1}, \sigma_{1}^{2}\right)$ corresponding to power samples observed in the presence of the PU signal. The set $\mathbf{P}$ was processed with the different methods considered in this work. For the FCME method, the value of the parameter $T_{C M E}$ was calculated based on (10) to provide CSSR $=1 \%$, which for the considered scenario $\left(N=100\right.$ and $\left.K=10^{5}\right)$ yields $T_{C M E}=1.2473$. For the general and proposed EM methods (both with $M=1$ ), the set of estimates $\boldsymbol{\theta}$ was initialised using the $k$-means algorithm following the principle described in Section IV-A, while the log-likelihood tolerance threshold in (16) was set to $\varepsilon=10^{-10}$. For all methods, the accuracy was evaluated by comparing the estimated noise floor with the true value. The experiment was repeated 500 times with different random sets $\mathbf{P}$ to ensure statistical reliability.

Fig. 1 shows the relative error of the estimated noise floor as a function of the SNR for the different methods considered in this work. As it can be appreciated, the Otsu method can provide an accurate estimation only under high SNR conditions (for SNR values greater than $-5 \mathrm{~dB}$ ). As discussed in Section III, under high SNR conditions the power samples drawn from $\mathcal{N}\left(\mu_{0}, \sigma_{0}^{2}\right)$ and $\mathcal{N}\left(\mu_{1}, \sigma_{1}^{2}\right)$ are sufficiently apart and a perfect classification of the observed power samples into both sets is possible. However, as the SNR decreases and both distributions overlap, a reliable classification is not possible and the estimation accuracy commences to degrade. As appreciated in Fig. 1, the accuracy of the Otsu method degrades for SNR values below $-5 \mathrm{~dB}$ and becomes particularly inaccurate for very low SNR, even though in the region of very low SNR all observed power samples are in practice noise-only. This is an inherent consequence of the criterion employed by the Otsu method, which sets the threshold somewhere in the middle of the observed power range, which divides the set into two groups (even when all samples are noise-only). As a result, this method provides the worst accuracy under low SNR. The other threshold-based classification method (i.e., the FCME method) does not suffer from this problem under low SNR as it rightly increases recursively the threshold until all power samples are correctly classified as noise-only; consequently, it can provide a very accurate estimation of the noise floor under low SNR conditions. The same applies to FCME in the case of high SNR, where a perfect classification is possible as described above. However, the accuracy of FCME is rather poor in the region of intermediate SNR values, approximately in the interval $[-14 \mathrm{~dB},-4 \mathrm{~dB}]$, where the samples from $\mathcal{N}\left(\mu_{0}, \sigma_{0}^{2}\right)$ and $\mathcal{N}\left(\mu_{1}, \sigma_{1}^{2}\right)$ overlap and a threshold-based classification is not reliable. On the other hand, the EM methods consistently provide a nearly perfect estimation in the region of medium/high SNR values. The accuracy suffers some degradation as the SNR decreases, however to different extents. The accuracy of the general EM method begins to degrade at an SNR of $-7 \mathrm{~dB}$ and deteriorates significantly until it becomes very inaccurate under low SNR conditions. On the other hand, the proposed EM method provides a virtually perfect estimation for SNR values above $-10 \mathrm{~dB}$ (i.e., $3 \mathrm{~dB}$ 


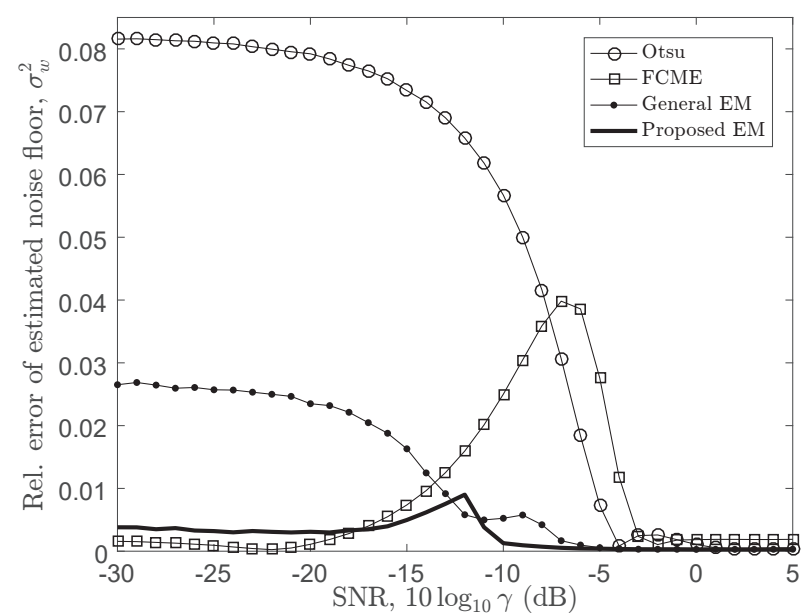

Fig. 1. Relative error of the estimated noise floor.

gain with respect to the general EM method); below this point the accuracy degrades slightly, however still remains within limits of very high accuracy. In particular, the worst estimation accuracy for the proposed EM method is observed at an SNR of $-12 \mathrm{~dB}$ where the relative error is only $0.009(0.9 \%)$, which for a noise floor of $-104 \mathrm{dBm}$ corresponds to an absolute power estimation error of $0.04 \mathrm{~dB}$. For SNR values below $-15 \mathrm{~dB}$ the relative error reduces to $0.003(0.3 \%)$, which for a noise floor of $-104 \mathrm{dBm}$ corresponds to an absolute power estimation error of $0.013 \mathrm{~dB}$. Only the FCME method provides a more accurate estimation of the noise floor under low SNR conditions, however the FCME estimation accuracy is poor for certain intermediate SNR values. Overall, only the proposed EM method can consistently provide an accurate estimation of the noise floor over the whole range of SNR values.

Sometimes the CR receiver may be more interested in the distribution of the noise power samples than their average value (for example, to set the decision threshold of an energy detector for a given probability of false alarm). Fig. 2 shows the accuracy of the estimated distribution of noise power samples, $\mathcal{N}\left(\mu_{0}, \sigma_{0}^{2}\right)$, in terms of the Kolmogorov-Smirnov (KS) distance between the estimated and true distributions. The trend of the KS distance is very similar to that of the relative error shown in Fig. 1. Only the proposed EM method provides an accurate estimation over the whole range of SNR values, with a worst-case error of $3.6 \%$ observed at an SNR of $-12 \mathrm{~dB}$ and a stable estimation error of $1.5 \%$ for SNR below $-15 \mathrm{~dB}$, which represents an excellent level of accuracy.

Fig. 3 shows the average computation time of each estimation method as a function of the SNR. While thresholdbased classification methods have the lowest computational cost, their accuracy is very limited as shown in Figs. 1 and 2. On the other hand, the EM methods provide a higher estimation accuracy at the expense of a higher computational cost as it can be appreciated in Fig. 3. Of particular interest is the appreciation that, under low SNR conditions, the proposed EM method not only provides a significantly higher accuracy than the general EM method (Figs. 1 and 2) but also does

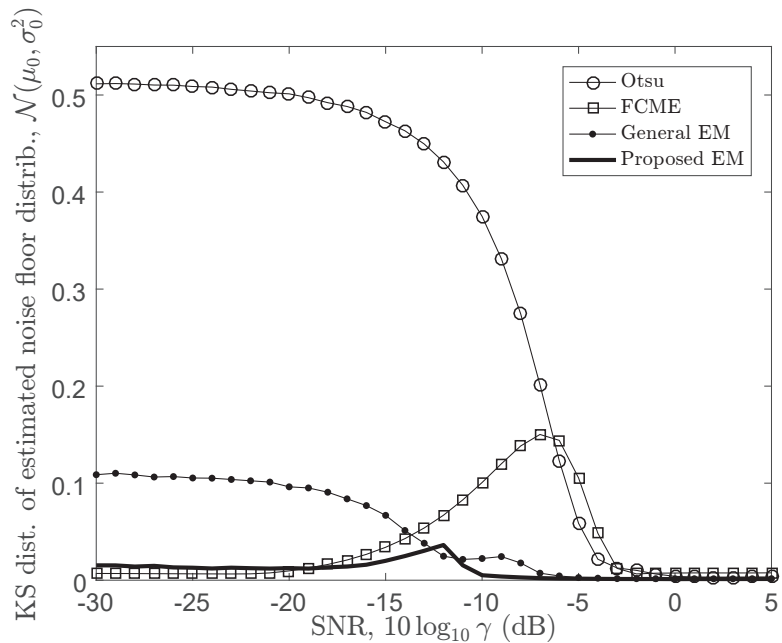

Fig. 2. Kolmogorov-Smirnov distance of the estimated noise floor distribution.

so at a lower computational cost (Fig. 3). Concretely, for the particular evaluation conditions considered in this work, the average computation time is reduced by a factor of three, from 9 seconds (general EM method) to 3 seconds (proposed EM method). This can be ascribed to the immediate calculation of $\sigma_{m}^{2}$ from the estimated $\mu_{m}$ based on (17) that the proposed EM method performs as opposed to the iterative estimation based on (14) that the general EM method carries out, which increases the number of iterations required until the algorithm converges. While the important aspect of relevance in Fig. 3 is the relative time difference among estimation methods, from a practical implementation point of view it is worth mentioning that these absolute computation times were obtained with a simulation code that was not optimised for performance and using a generic-purpose computer with an off-the-shelf processor Intel Core i3-6100 at 3.7 GHz. A realistic implementation based on specialised components (e.g., FPGA, ASIC or SoC) and optimised for performance would be expected to run the algorithm within significantly lower execution times.

Finally, as pointed out in Section IV, the EM methods provide an estimation of the complete set of parameters of the Gaussian mixture, including not only the noise floor $\left(\mu_{0}\right)$ but also the channel duty cycle $(\Psi)$ and the SNR $(\gamma)$, which can be obtained based on (7). The ability of these methods to estimate these other parameters of practical relevance was investigated as part of this research as well. While the SNR estimation resulting from the application of the EM methods was observed to be inaccurate, the obtained duty cycle estimation was actually very accurate. Fig. 4 shows the estimated duty cycle for the EM methods along with the estimation obtained with the threshold-based classification methods (calculated as the fraction of power samples above the threshold). Thresholdbased methods can provide an accurate estimation of the duty cycle for SNR values greater than $-3 \mathrm{~dB}$ (FCME) and $-5 \mathrm{~dB}$ (Otsu), while EM methods provide a better sensitivity with accurate estimations down to $-7 \mathrm{~dB}$ (general EM method) and $-10 \mathrm{~dB}$ (proposed EM method). As it can be appreciated, the 


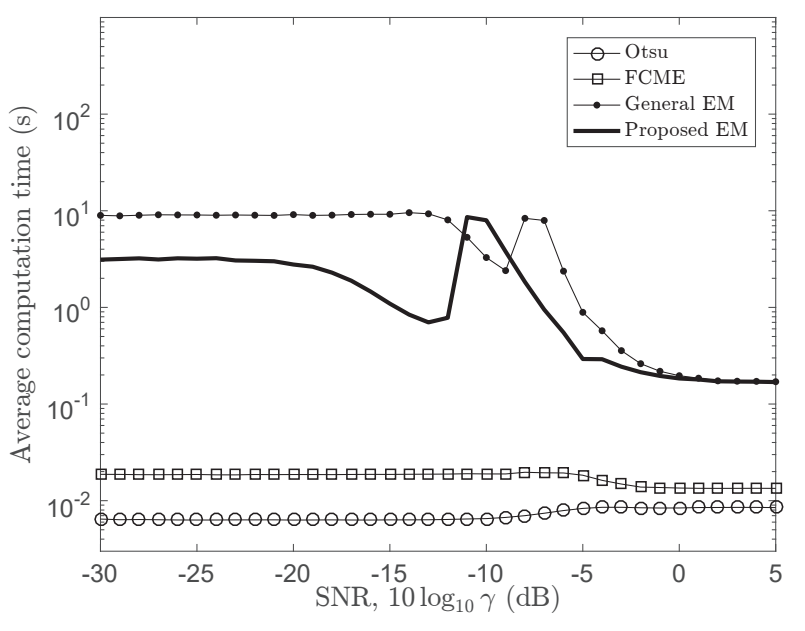

Fig. 3. Average computation time.

proposed EM method provides the best sensitivity, with gains of $3 \mathrm{~dB}$ with respect to the general EM method and 5-7 dB with respect to the threshold-based classification methods.

\section{CONCLUSions}

CR devices can benefit significantly from effective methods that enable an accurate estimation of the noise floor. Most existing methods are aimed at isolating a subset of noiseonly power samples based on a certain decision threshold. However, this type of approaches are in general unable to provide accurate estimations, in particular under low SNR where the PU signal level is comparable to the noise floor of the CR device. By modelling the distribution of observed power samples as a Gaussian mixture, this work has explored the possibility to estimate the noise floor by fitting such model to empirical observations. While the general EM method commonly used to this end suffers from similar inaccuracy issues, a modified version that takes into account the relation among the parameters of the Gaussian mixture can provide accurate estimations. The proposed EM method not only consistently provides a highly accurate estimation of the noise floor in the presence of PU signals over the whole SNR range but does so at a much lower computational cost than the general EM method. The proposed EM method can also provide a nearly perfect estimation of the channel duty cycle over a larger range of SNR values compared to threshold-based approaches.

\section{ACKNOWLEDGEMENTS}

This work was supported by British Council under UKIERI DST Thematic Partnerships 2016-17 (ref. DST-198/2017). The work of J. Lehtomäki was supported by the Academy of Finland 6Genesis Flagship (grant 318927) and Infotech Oulu. The work of $\mathrm{K}$. Umebayashi was supported in part by the MIC/SCOPE under Grant 165003006, and in part by the JSPS KAKENHI under Grant JP15K06053 and Grant JP15KK0200.

\section{REFERENCES}

[1] M. López-Benítez, “Cognitive radio,” in Heterogeneous cellular networks: Theory, simulation and deployment.Cambridge Univ. Press, 2013.

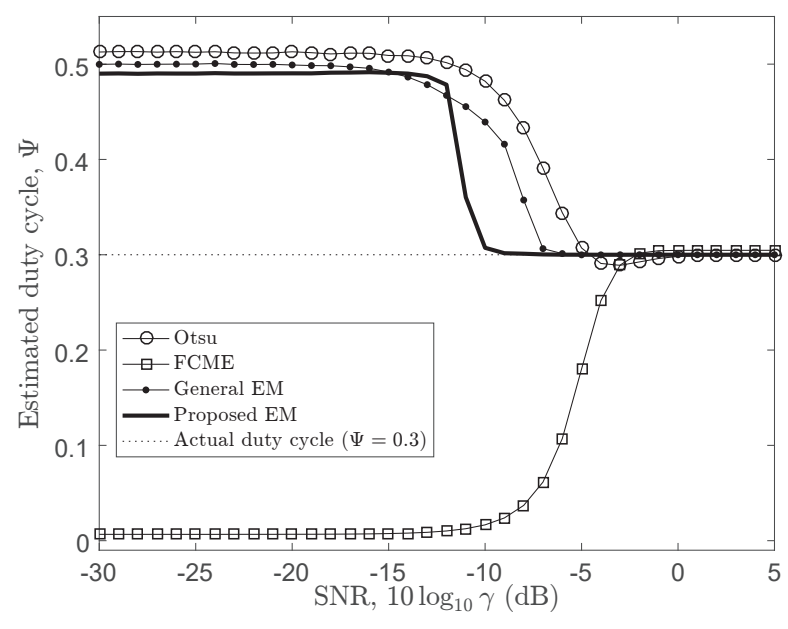

Fig. 4. Estimated duty cycle.

[2] T. Yücek and H. Arslan, "A survey of spectrum sensing algorithms for cognitive radio applications," IEEE Comms. Surveys \& Tutorials, vol. 11, no. 1, pp. 116-130, First Quarter 2009.

[3] J. Lehtomäki, M. Juntti, and H. Saarnisaari, "CFAR strategies for channelized radiometer," IEEE Signal Processing Letters, vol. 12, no. 1, pp. 13-16, Jan. 2005.

[4] M. López-Benítez and J. Lehtomäki, "Energy detection based estimation of primary channel occupancy rate in cognitive radio," in Proc. IEEE Wireless Comms. \& Netw. Conf. (WCNC 2016), Apr. 2016, pp. 306-311.

[5] R. Tandra and A. Sahai, "SNR walls for signal detection," IEEE Journal of Selected Topics in Signal Procesing, vol. 2, no. 1, pp. 4-17, Feb. 2008.

[6] D. Torrieri, "The radiometer and its practical implementation," in Proc. Military Comms. Conf. (MILCOM 2010), Oct. 2010, pp. 304-310.

[7] M. López-Benítez and F. Casadevall, "Methodological aspects of spectrum occupancy evaluation in the context of cognitive radio," European Trans. Telecomms., vol. 21, no. 8, pp. 680-693, Dec. 2010.

[8] _ "On the spectrum occupancy perception of cognitive radio terminals in realistic scenarios," in Proc. 2nd Int'l. Workshop on Cognitive Information Processing (CIP 2010), Jun. 2010, pp. 99-104.

[9] M. Ready, M. Downey, and L. Corbalis, "Automatic noise floor spectrum estimation in the presence of signals," in Proc. 31st Asilomar Conf. on Signals, Systems \& Computers, Nov. 1997, pp. 877-881.

[10] N. Otsu, "A threshold selection method from gray-level histograms," IEEE Trans. Syst., Man, \& Cyber., vol. 9, no. 1, pp. 62-66, Jan. 1979.

[11] D. Datla, A. M. Wyglinski, and G. J. Minden, "A spectrum surveying framework for dynamic spectrum access networks," IEEE Trans. Vehic. Tech., vol. 58, no. 8, pp. 4158-4168, Oct. 2009

[12] H. Saarnisaari, P. Henttu, and M. Juntti, "Iterative multidimensional impulse detectors for communications based on the classical diagnostic methods," IEEE Trans. Comms., vol. 53, no. 3, pp. 395-398, Mar. 2005.

[13] A. Agarwal, A. S. Sengar, and S. Debnath, "A novel noise floor estimation technique for optimized thresholding in spectrum sensing," in Proc. Int'l. Conf. Advances in Computing, Comms. and Informatics (ICACCI 2017), Sep. 2017, pp. 607-611.

[14] J. J. Lehtomäki, R. Vuohtoniemi, K. Umebayashi, and J.-P. Mäkelä, "Energy detection based estimation of channel occupancy rate with adaptive noise estimation," IEICE Trans. Comms., vol. E95-B, no. 4, pp. 1076-1084, Apr. 2012.

[15] B. Selim, O. Alhussein, S. Muhaidat, G. K. Karagiannidis, and J. Liang, "Modeling and analysis of wireless channels via the mixture of Gaussian distribution," IEEE Trans. Vehic. Tech., vol. 65, no. 10, pp. 8309-8321, 2016.

[16] J. J. Lehtomäki, J. Vartiainen, M. Juntti, and H. Saarnisaari, "CFAR outlier detection with forward methods," IEEE Transactions on Signal Processing, vol. 55, no. 9, pp. 4702-4706, Sep. 2007.

[17] M. Abramowitz and I. A. Stegun, Handbook of mathematical functions with formulas, graphs, and mathematical tables, 10th ed. Dover, 1972.

[18] A. P. Dempster, N. M. Laird, and D. B. Rubin, "Maximum likelihood from incomplete data via the EM algorithm," Journal of the Royal Statistical Society, Series B, vol. 39, no. 1, pp. 1-38, 1977. 